\title{
A Systematic Review of the Selected Evidences on the Effectiveness of Inter-professional Education (IPE) in Developing Interprofessional Learning Environment
}

\author{
Said Nasser Al Harthy ${ }^{1}$, Najla Al Subhi ${ }^{2}$, Cyruz Tuppal ${ }^{3}$, Mark Donald Reñosa ${ }^{4}$
}

\begin{abstract}
Purpose: This systematic review aimed to provide available evidence in determining for the effectiveness of IPE as a modality in developing interprofessional learning environment for healthcare students.

Methods: The computerized searches from 2009-2015 in ten electronic databases were performed. Two independent reviewers were consulted to assess the eligibility, level of evidences and methodological quality in each study.

Result: nine out of ten studies were retrieved. These studies include (a) two RCT studies; scored eight and seven out of eight, (b) Three quasi-experimental pretestposttest design; scored seven, six, and six out of eight, (c) Three controlled before and after study; scored six, six, five and five out of eight, and (d) one controlled longitudinal; scored six out of eight respectively.

Conclusion: Based on the systematic review, evidence showed that IPE was effective in building strong interprofessional learning environment. On the other hand, the authors recommend considering conduct of similar systematic review grounded on IPE with larger sample size within the health allied discipline.
\end{abstract}

Keywords: inter-professional education, nursing education, nurses, systematic review

\footnotetext{
${ }^{1}$ Faculty, Oman Specialized Nursing Institute, Oman. Email: saidbro75@gmail.com

${ }^{2}$ Faculty, Oman Specialized Nursing Institute, Oman.

${ }^{3}$ Faculty, St Paul University Philippines, Philippines.

${ }^{4}$ Science Research Specialist, Research Institute for Tropical Medicine, Philippines.
} 


\section{BACKGROUND}

Healthcare professionals are becoming more grounded on team-based practice. Such practice builds and supports teamwork that further improves communication skills, shared decision making, problem solving, competencies, patient care and novelty contribution from each discipline (Peduzzi, et al., 2013;Barr, et al., 2005). On the contrary, uniprofessional approach still emanates within the healthcare education, where learners learn and practice specific outcome and activities of knowledge, skills, and attitude that relate to students in a same profession and isolated from other professions (Thistlethwaite \& Moran, 2010; Peduzzi, et al., 2013).

For instance, nursing, medical and allied health care students are entering their professional courses with a stereotyped idea about their own profession and competencies. Which it is effective in forming a specific professional identity, rules, responsibilities, develop specialist knowledge, creates power and status which is essential for health care system (Becker, Hanyok \& Walton-Moss, 2014). On the other hand, it is believed that this uniprofessional academic is not to prepare students for professional teamwork that is required in current healthcare environment (Inuwa, 2012). On that note, the health education system should innovate and look for better ways to prepare health care learners on their roles on the professional team in order to improve the competencies, hence should be included in curriculum.

Up to date, the clinical governance and healthcare institutions have recognized the importance of removing the professional boundaries in relation to rules and responsibilities between health care professionals and requires to share competencies, communication and knowledge. Patient care and needs are best met by collaborative work among the health care providers (Inuwa, 2012). Lumague, etal., (2006) have recommended that health care providers should work in interprofessional team as it improves the communication skills and sharing of knowledge.

In order to prepare healthcare profession to work as effective team, it is believed that this can be achieved by Inter-professional Education (IPE). The main idea of IPE is to ensure that health care learners entering the workforce, should be an effective teamplayer and team-based care provider. The concept of IPE is not new for educators, healthcare professions and researchers, as has gained global support as an approach that could be addressed in this times of contemporary healthcare challenges. WHO (1988),supported the idea of IPE as a method that enhances interprofessional teamwork and collaboration and even provided an outlined IPE content and developed core competencies for effective teamwork.

Several countries are actually implementing this approach, in fact, Bandali, et al., (2010), espoused that the Health Council in Canada has recommended that IPE subject should be offered in all universities health science program. In United Kingdom, the Department of Health and Quality Assurance Agency (2006) addressed IPE as compulsory in all pre-registration training in health and social care. It is also good to note that, the Institute of Medicine (2001) in the United States published a paper recommending that health care professionals should be educated in taking patient care as an interdisciplinary team. The Department of Health and Ageing (2008) in Australia 
also reported the importance of interdisciplinary learning in their report on their national primary health care strategy. In Japan, Takahashi (2007) has reported the importance of IPE in bringing a suitable structure for interprofessional health care practice expected to meet the contemporary health-care. In addition, The Japan Inter Professional Working and Education Network (JIPWEN) was established to support IPE and practice in 2008.

Several studies also confirmed its importance and usage. Priddis \& Wells (2011) asked senior staff regarding the implications of IPE and the findings indicated that knowledge, skills and confidence increased as well as the communication skills were all improved. Also, Lumague et al., (2006) have conducted a study among nine students from seven different health care profession "medicine, nursing, occupational therapy, pharmacy, physiotherapy, social work and speech language pathology on the purpose of interprofessional collaboration, results showed an increase and improvement of communication skills and enhancement of knowledge on the different roles for each discipline in health care team which fosters respect. They found that all participants valued the importance of interprofessional teamwork in patient care and the education should enable the development of skills, behavior and attitude for interprofessional collaboration and improving communication skills.

Moreover, McNair (2005), discussed on the literature review that was held by National Institute of Clinical Studies (2003), on factors that support high performance in health care. The literature review found that "the potential of health care team was not being realized because of lack of effective communication and team working practice (NICS,2003, p.15). There is evidence of the effectiveness of interprofessional teamwork" as it improves communication, as it is cost effective, focuses on patientcenteredness care, heightens level of respect between the members, there was a clear understanding of the roles and skill and sustainable work satisfaction (McNair, 2005).

However, even though international support and recommendation for IPE as an important area for health professional education, there were lack of systematic evidences of its effectiveness (Braithwaite, et al., 2007, and Lapkin, Levett-Jones, \& Gilligan, 2013). Although, there were number of research studies support on IPE, the methodological rigour were not strong and most of the studies were on students and not compared with uniprofessional education. Moreover the concept if IPE is still not clear, as there are number of different definition (Braithwaite \& Travagila, 2005), objectives, strategies of implementing it, venues and duration (Inuwa, 2012; Reeves, et al., 2013).

\section{OBJECTIVE}

The aim of this review was to comprehensively search and systematically appraise for the best and recent studies into the effectiveness of IPE in promoting competencies compared with uniprofessional for both under and post graduate health care students and provide synthesis of the findings.

\section{METHODS}

In order to assess the effectiveness of IPE intervention compare with traditional education a systematic review of the literature was conducted, searching for both 
published and unpublished publications studies, limited to English language and published between 2009-2014.

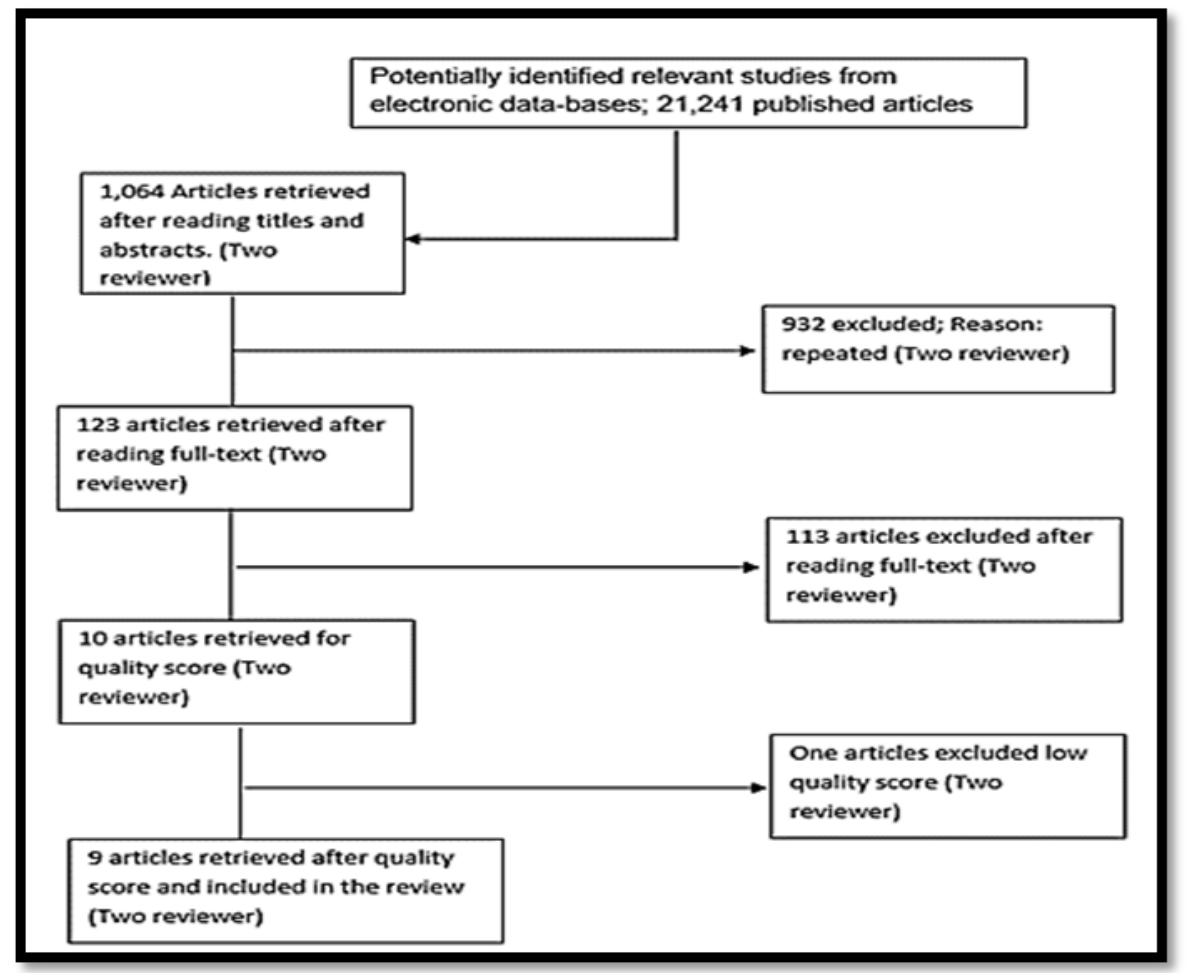

Figure 1. Flowchart of articles selected from search and appraisal results

\section{Studies and Scores}

The Joanna Briggs Institute Library of Systematic Reviews(JBI) appraisal tool was modified as Questions related to blinding and concealing of the reviewers. Referring to JBI's (2014), quality score and data extraction, nine studies published between 2009 and 2014, mixed quantitative studies, varying in approach, used convenience sampling, differ in scores and in different countries were included for the review. Two RCT studies by (Nango \&Tanaka, 2010; Just, Schnell, et al.,2010), three quasi-experimental pretest-posttest design (Bradley, Cooper, \&Duncan, 2009; Delunas \& Rouse, 2014; Scherer, et al., 2013), three controlled before and after study (Anderson, et al., 2009; Ateah, et al., 2011; Ritchie, Dann \& Ford, 2012) and one controlled longitudinal (McFadyen, et al., 2010) respectively.

The majority of participants included in the studies were from undergraduate nursing and medical students (Ateah, et al., 2011; Bradley, et al., 2009; Delunas \& Rouse, 2014; Just, et al., 2010; Nango \&Tanaka, 2010; McFadyen, et al., 2010; Scherer, et al., 2013). Other professions were:occupational therapy, physical therapy, dental hygiene, pharmacy and dentistry postgraduate physiotherapy, health and social care student, radiography, podiatry, prosthetics, orthotics. One study included master's students from pharmacy and nursing (Nango \&Tanaka, 2010) although one study (Anderson, et al., 2009) did not give the details of other profession that were included with medical students. The sample size of the participants in studies were ranging from 34 (Nango \& Tanaka, 2010) to 573 (McFadyen, et al., 2010), respectively. 


\section{Comparability and confounding}

Seven studies gave comparable details related to the participants at their entry such as age, sex (Ateah, et al.,2011; Bradley, et al., 2009; Delunas \& Rouse, 2014; Just, et al., 2010; McFadyan, et al., 2010, Nango \& Tanaka, 2010; Ritchie, et al., 2012). However, other studies added other method of comparison such asPre-IPE experience (Bradley, et al., 2009) or experience health care before being students (Delunas \& Rouse, 2014). In these studies, the participants in both group were relatively homogenous at entry. There were two studies that did not give details of entry comparability (Anderson, et al., 2009; Scherer, et al., 2013).

Likely, the studies have showed that the work of minimizing the effect of the confounders' variables was by making the participants more similar in studies. In addition, there were no differences in how the experimental and control group were treated as all studies defined the process by which they delivered IPE but the approach and setting that they have used were different from study to study.

\section{Outcome measures instruments}

The instrument that were used in measuring objectively or self-reports of the main educational outcome competencies toward IPE were thought to be reliable and valid instruments. Such as Readiness for Interprofessional Learning Scale (RIPLS), Student Stereotypes Rating Questionnaire (SSRQ), Leadership Behavior Description Questionnaire (LBDQ), Emergency Team Dynamics (ETD), Resuscitation Team Task (RTT), Jefferson scale of attitudes Interdisciplinary Education Perception Scale (IEPS). However, three studies developed their own tool and failed to give details about reliability and validity testing (Anderson et al., 2009; Just et al., 2010; Nango \& Tanaka 2010).

\section{RESULT}

\section{Description of studies}

For the Randomized Control Trials studies by Just, et al (2010) and Nango and Tanaka (2010), they both used a computer random number generation for the randomization. However they were differ in the method of applying the IPE and the outcome. In the study of Just, et al., (2010), they assessed the effects of an IPE course on nursing and medical students' behavior on the course of interprofessional communication skills and patient care. They were $(\mathrm{N}=40)$ third year undergraduate nursing and medical students in the study.The participants were randomly assigned into two groups, the experimental group $(n=20)$ and the control group $(n=20)$. All participants were given a case based on palliative care for elderly patient. The experimental group $(n=20)$ were taught the interprofessional core competencies utilizing different learning strategies such as roleplay, power-point presentation, case studies, references articles, discussion and reflection. In contrast the control group $(n=20)$ where not taught by tutors, they were given written material (text, slides and paper-cases) which were used by the experimental group. The control group were also told to study silently and prevent interaction with others and this was regulated by their supervisor. 
On the other hand, Nango and Tanaka (2010) utilized participants who were postgraduate master students in the profession of pharmacy and nursing and fifth year medical students in evaluating the effectiveness of IPE on clinical decision making by using problem based learning. Medical students $(\mathrm{N}=20)$ were randomly assigned to either interprofessional group that consist of master students from nursing $(\mathrm{N}=9)$ and pharmacy $(\mathrm{N}=8)$ or a uniprofessional group medical student only. Both groups have received the same intervention as they have attend the two day PBL program focusing on Evidence Based Medicine (EBM), all participants have received an original text book explained the concept of EBM,15 minutes lecture explaining the four steps of EBM, they were also tasked to work on case scenarios. The participants were separated into groups and had a small group discussion for 90 minutes for presenting core information about the case and formulating a clinical question "PICO" statement.

The outcome measuring tool were also differed. Just et al (2010) used a grid for the measurement tool suitable for transcribing the video simulation material evaluating "initiation, interruptions, speaking time, and exchanged information items", while Nango and Tanaka (2010) utilized visual analog scale, written test, information cards and 5-point Likert scale questionnaire. In Just et al (2010), there were statistically significant improvement in number of exchange information items for both group post intervention. Whereas, there were no statistical significant differences in initiation, interruptions and speaking time. Meanwhile, in the study of Nango and Tanaka (2010),there were no statistical differences in clinical epidemiology knowledge, additional patient information and students' evaluation of PBL program between the groups. However, the PBL program with nurses, pharmacy and medical have significantly affect the clinical decision making by medical students.

For the three quasi-experimental studies (Bradley,et al., 2009; Delunas \& Rouse, 2014; Scherer, et al., 2013). The participants were undergraduate nurses and medical students in all three studies, however they were differed in numbers of participants, aim and outcome measurements. Bradley, et al., (2009) aimed to identify the effects of IPE experience on fifty-three (53) second year nursing and medical students' attitude, leadership, team-work and performance of resuscitation skills, in two different university. Initially seventy-one randomly selected students were allocated to either the uniprofessional (control) group or the Interprofessional (experimental) group and they were blinded to study objectives. Same intervention was implemented in both groups every day on Intermediate Life Support (ILS) programme by a life support instructor. The differences of students attitudes toward IPE were evaluated at three points in time: before and after each course and 3-4 months laterbyRIPLS. The result indicated that the scores on the RIPLS subscales of teamwork and collaboration and professional identity scores increased significantly after the ILS programme for interprofessional group however fell back to pre-test level after 3-4months. Nursing students compared with medical students had significantly higher scores on the roles and responsibilities subscales at all three data collection points. The results did not show any other significant differences across the three sets of RIPLS scores and performance for any of the participants. 
Quasi-experimental design by Delunas and Rouse (2014) with pre and post-test measures the effects of IPE program integrated into existing curriculum on nurses and medical students' attitude about communication and collaboration. The participants consisted of first-year medical students $(n=18)$ and junior nursing students $(n=17)$ were assigned in interprofessional (Intervention) and second year medical students $(n=18)$ and junior nursing students $(\mathrm{n}=21)$ as a control group. Both participants completed the time two (2) data collection, whereas for the time one (1), the researchers were unable to select the data for the second year medical students due to unforeseen logistical issues, therefore, data from this group were not viewed as control data. Jefferson Scale of Attitude (JSA) toward physician-nurse collaboration was used to measure attitude about communication and collaboration and the Baggs Collaboration and Satisfaction about Care Decisions tool, at two point times, before and after the project of Health Care Team.

The score items in attitude toward communication and collaboration differed significantly between the medical and nursing groups at the begging of the study, with medical students having significantly less positive attitude toward interprofessional communication and collaboration. At time two, again the medical students had significantly less positive attitude.

Scherer, et al., (2013) conducted a quasi-experimental study using a pre-post test design to investigate the effect of IPE on experimental nurses $(\mathrm{N}=48)$ and medical students $(\mathrm{N}=23)$ compared with control group nursing students only $(\mathrm{n}=37)$ on knowledge, confidence, readiness and attitudes toward interprofessional learning, teamwork and collaboration. Nursing students were randomly assigned to either the interprofessional experimental or intraprofessional control simulation group, whereas, medical students were enrolled in experimental group. Both group were provided the information about the study, had prior exposure to simulation and completed didactic instruction on the management of cardiac patients; however it was the first time to participate in interprofessional scenarios. The researchers used multi-method evaluation, knowledge test, the confidence scale the RIPLS and the Attitude Towards Health Care Teams Scale (ATHCTS). Students did a pre-test immediately after check in at the simulation centre and post-test after the debriefing. Results indicated that, students in interprofessional simulation group score on knowledge; the three RIPLS subscales: teamwork and collaboration, professional identity and roles and responsibilities; and two subscales of the ATHCT: quality of care/ team process improved significantly in comparison with the control group.

For the four controlled before and after study: Anderson, et al., (2009) conducted a controlled pre and post-study. Assessing medical students' knowledge gained perceptions of IPE after being in either a uniprofessional or interprofessional workshop. Undergraduate medical and other profession students (not known the other profession) $(\mathrm{N}=199)$, control group consist of medical students only learn $(n=58)$ and the experimental group consist of medical students and the other profession $(n=141)$. For the intervention, it consists of a one-day patient safety workshop based on a film depicting an elderly woman who is admitted in rehabilitation ward. The film was played in sections so the students could focus on the learning themes of situational awareness, 
communication, leadership and empowerment and to analyze key safety issues and teamwork. Students were given relevant material for each section. By the end of the day, students used root cause analysis and personal analysis reflecting their competencies. Data were collected by knowledge acquisition questionnaires. The result indicated that, although both group achieved increased their knowledge across the learning outcome, and for the key learning themes the medical students in the uniprofessional group achieved significantly higher scores than those in interprofessional group.

On the other hand, the study of Ateah, et al., (2011) utilized a modified experimental pre-test post-test design to provide evidences about the effectiveness of IPE interventions on pre-licensure students by comparing knowledge, attitudes, skills and values in promoting collaborative patient-centered care. Total participants were fiftyone (51) from seven health programs at the University of Manitoba in Canada. Eighty five percent of the participants were randomly assigned into one of three groups of interprofessional learners and some self-selected into certain groups; a control group $(\mathrm{n}=17)$, and two experimental group; an education-only intervention group $(\mathrm{n}=16)$ and interprofessional immersion experience intervention group $(n=18)$. On the first day all three groups attended a 30-minute orientation to the research project and completed the survey by Student Stereotypes Rating Questionnaire (SSRQ). Then, the two experimental group continued the IPE intervention that lasted for a two and a half day education. The intervention included definition of IPE for collaborative patient centered practice, interactive sessions, didactic learning activities, presentations, group exercise, brain storming and reflection. In addition, patients with chronic health condition provided two presentation about their experience with health care professional. After the education intervention, the inter-professional immersion experience, intervention group were divided into four or five groups and sent to different clinical sites for the collaborative practice; where they have observed the interprofessional interactions and collaborative patient/client care and shadowed health professionals and other staff. On the last day of the IPE immersion experience, each groupfrom the different site has presented a general reflection, programme-specific reflections and a case presentation and they completed the SSRQ. After four to five months from the last-day of IPE session the SSRQ was sent to all participants to evaluate the outcome of the IPE. To suffice with, all participants were evaluated by SSRQ after the introduction of the IPE and after four months post IPE immersion experience. The results indicated a significant increase in scores of all traits between the first and second survey, and between the first and third survey (after four months)for the participants experienced the IPE classroom sessions and site immersion experience.

A study by Ritchie, et al., (2013) aimed to evaluate the understanding of roles and responsibilities and attitudes toward interprofessional learning over a two years period. The participants consist of Bachelor of Oral Health and Bachelor of Dental Science students with a total sample size of 186. The students in the control group ( $n=93)$ was enrolled into a Traditional, Discipline-specific programme (TRAD) whereas the experimental group $(n=93)$ experienced the Integrated learning programme (INT) with combined courses for oral health and dental science students. The intervention, for the experimental group (INT) students were introduced to the differences and 
complementarity of roles for dentists and oral health therapists and the scope of practice were highlighted in seminars, case-based learning exercises and assessment tasks and were required to work in mixed teams and to maximize socialization and interprofessional learning. Both cohort of TRAD and ITN, participated in a single day activity (a faculty-wide interprofessional learning activity) as an introductory day to teamwork, problem solving, collaboration and conflict resolution and also introduced to the concept of Health Care Team. The RIPLS was used to all participants to measure the outcome. The results indicated that following the completion of one year of learning, both INT and TRAD cohorts showed similar level of readiness for shared learning and there were no significant Results.

Moreover, McFadyen, et al.,(2010) used a controlled longitudinal study to assess the impact of IPE intervention over four years, on the attitudes and perceptions of preregistration health and social care students with respect to IPE. The participants consist of students from six professions: Nursing, Occupational Therapy, Podiatry, Prosthetics \& Orthotics, Physiotherapy and Radiography with a total sample sizeof 573; where $(\mathrm{N}=260)$ in control group who commenced their pre-registration program in 2003 and $(\mathrm{N}=313)$ in the experimental group commenced in 2004. In terms of intervention, the control group received uni-professional education whereas the experimental group received an IPE, consist of combination of two specific elements, a 20 credit point formal module with themed days during each academic year. The module in each year covered in 24 weeks covering a keynote lecture followed by mixed interprofession seminar discussion. For the themed days, it covered topics that were relevant to all profession such as communication, topics were chosen by the IPE project group members, this occurred once during each semester for the duration of the study. The outcome were measured through the adapted version of the RIPLS and the IEPS. For the RIPLS subscales, the results indicated that the mean scores of three subscales of teamwork and collaboration, negative professional identity, positive professional identity, and role and responsibilities exceed $75 \%$ of the maximum. For the IEPS, the results illustrated that, there were a positive attitude effects in two subscales of competency and autonomy and perception of the actual cooperation toward IPE in the experimental group.

\section{DISCUSSION}

This systematic review aimed to examine the effectiveness of IPE in promoting competencies for the health care students. For a variety of reasons, it is difficult to draw a firm conclusion from the identified studies. The reviewers applied all the systematic review process stages, which resulted in only nine publications from 2009 to 2014 being included. Two of the included publications were studies involving RCTs, three quasiexperimental; four controlled before and after study and one a controlled longitudinal study.

\section{Change in students' attitude}

There was evidence of changes in health care students' attitudes toward IPE in six of the studies reviewed (Ateah, et al., 2011; Bradley, et al., 2009; Delunas \& Rouse, 2014; McFadyen, et al., 2010; Ritchie, et al., 2013 and Scherer, et al., 2013). However, the study by Bradley, et al., (2009) explicated that the level of attitude for the 
interprofessional group have returned to pre-test level after 3-4 months. This is important result as the main idea of IPE is that preparing health professional students for collaboration and teamwork. By providing time for professional practice and other variables that comes after the educational intervention period, it is essential to conduct studies that totally explore the generalisability of IPE competencies to clinical practice.

\section{Learning Outcomes}

For the learning outcomes of IPE, four studies (Anderson, et al., 2009; Just, et al., 2010; Nango \& Tanaka, 2010; Scherer, et al., 2013) reported mixed results. The study by Anderson, et al., (2009) reported that knowledge score in the control were higher than in the experimental group. Just, et al., (2010), on the other hand, reported that both group control and experimental group increased their scores in patient care objectives. Moreover, the interprofessional communication style had a moderate improvement. Nango and Tanaka (2010) reported only medical students improved their ability in clinical decision-making. Scherer, et al., (2013) reported the experimental group had a higher score on knowledge than the control group.

\section{IPE approach}

The reviewed studies differed in the way in which they implemented the intervention, number of participants, settings, and length of sessions. It is believed that IPE approaches may be difficult to implement in a same way as number of constrictions inherent in university programs. As each university offer different curriculum design, timetabling, number of students and resources in nursing, medical and allied health care programs and this argued to make IPE challenging to implement.

All of studies in this review had methodological shortcomings and most of them used convenience sampling and this limits the generalizability of findings. Moreover, there was insufficient power to detect the effect of the various intervention due to small sample size. There were no comparative data in order that changes in outcomes could be accurately identified. Also, most of the studies, the participants were not blinded and according to Polit and Beck (2012), this increase the potential for social desirability bias in participants' behavior. The validated assessment tools that used for measuring the attitude and perception were also limited.

\section{CONCLUSION}

The evidence that support the effectiveness of IPE in enhancing health care students' attitudes toward team work and communications skills is not strong enough. Having dearth of evidence, further studies of the same related topic should be espoused by more researchers from interprofessional spectrum.

\section{REFERENCES}

Anderson, E., Thorpe, L., Heney, D., \&Petersen, S. (2009).Medical students benefit from learning about patient safety in an interprofessional team. Medical Education, 43, 542-552.

Ateah, C., Snow, W., Wener, P., MacDonald, L., Metge, C., Davis, P., Fricke, M., Ludwig, S., \&Anderson, J. (2011). Stereotyping as a barrier to collaboration: 
Does interprofessional education make a difference? Nurse Education Today, 31(2), 208-213.

Bandali, K., Niblett, B., Yeung, T.P., \&Gamble, P., (2010). Beyond curriculum: embedding interprofessional collaboration into academic culture. Journal of Interprofessional Care,25(1), 75-76.

Barr,H. (2009). An Anatomy of continuing Interprofessional Education. Journal of Continuing Education in the Health professions, 29(3), 147-150.

Barr, H., Koppel, I., Reeves, S., Hammick, M., \& Freeth, D. (2005). Effective Interprofessional Education: Argument, Assumption and Evidence. Oxford: Blackwell.

Becker, K. L., Hanyok, L. A., \& Walton-Moss, B. (2014). The turf and baggage of nursing and medicine: Moving forward to achieve success in inter-professional learning and practiceeducation. Journal for Nurse Practitioners, 10(4), 240-244.

Bradley, P., Cooper, S., Duncan, F. (2009). A mixed-methods study of interprofessional learning of resuscitation skills. Medical Education, 43(9), 912-922.

Braithwaite, J., Westbrook, J., Foxwell, A., Boyce, R., Devinney, T., Budge, M., \&Robyn, C. (2007). An action Research protocol to strengthen system-wide interprofessional. BMC, 13(7), 144-153.

Braithwaite, J.\&Travaglia, J. (2005). Inter-professional learning and clinical education: an overview of the literature. Canberra: Braithwaite and Associates and the ACT Health Department.

Delunas, L. \& Rouse, S. (2014). Nursing and medical student attitudes about communication and collaboration before and after an interprofessional education experience. Nursing Education Perspectives, 36(2), 100-105.

Department of Health \& Quality Assurance Agency.(2006). Department of Health Phase 2 Benchmarking Project - Final Report. Department of Health, London and Quality Assurance Agency for Higher Education, Gloucester.

Department of Health and Ageing.(2008). Towards a National Primary Health Care Strategy. Department of Health and Ageing, Canberra.

Institute of Medicine. (2001). Crossing the Quality Chasm: A New Health System for the 21st Century. National Academy Press, Washington, DC.

Inuwa, I. (2012). Interprofessional Education (IPE) Activity amongst Health Sciences Students at Sultan Qaboos University: The time is now! Sultan Qaboos University Medical Journal, 12(4), 435-441.

Just, J.M., Schnell, M.W., Bongartz, M., \&Schulz, C. (2010). Exploring effects of interprofessional education on undergraduate students' behaviour: a randomized controlled trial. Journal of Research in Interprofessional Practice and Education.1(3), 182-199.

Lapkin, S., Levett-Jones, T.,\& Gilligan, C. (2013). A systematic review of the effectiveness of interprofessional education in health professional programs. Nurse Education Today, 33(2), 90-102.

Lumague, M., Morgan, A., Mak, D., Hanna, M., Kwong, J., Cameron, C., Zener, D., \& Sinclair, L. (2006). Interprofessional education: The student perspective. Journal of Interprofessional Care, 20(3), 246-253.

McFadyen, A.K., Webster, V.S., Maclaren, W.M., \&O'Neill, M.A. (2010). Interprofessional attitudes and perceptions: results from a longitudinal controlled 
trial of pre-registration health and social care students in Scotland. Journal of Interprofessional Care, 24(5), 549-564.

McNair, H. (2005). The case for educating health care students in professionalism as the core content of interprofessional education. Medical Education, 39(5), 456-464

Nango, E., Tanaka, Y. (2010).Problem-based learning in a multidisciplinary group enhances clinical decision making by medical students: a randomized controlled trial. Journal of Medical and Dental Sciences, 57(1), 109-118.

National Institute of Clinical Studies. (2003). Factors supporting high performance in health care organizations. Melbourne: NICS, Health Management Group La Trobe University.

Peduzzi, M., Norman, I., Germani, A., da Silva, J., \& Souza, G. (2013). Interprofessional education: training healthcare professionals for teamwork focusing on users. Revistada Escola de Enfermagem da USP, 47(4), 977-983.

Polit, D. \& Beck, C. (2012).Nursing research: Generating and Assessing Evidence for Nursing Practice. $\left(9^{\text {th }}\right.$ ed.) Philadelphia: Lippincott Williams and Wilkins.

Priddis, L. E., \& Wells, G. (2011). Innovations in interprofessional education and collaboration in a West Australian community health organisation. Journal of Interprofessional Care, 25(2), 154-155.

Reeves, S., Zwarensten, M., Goldman, J., Barr, H., Freeth, D., Koppel, I. (2010). The effectiveness of interprofessional education: Key findings from a new systematic review. Journal of Interprofessional Care, 24(3), 230-241.

Reeves, S., Perrier, L., Goldman, J., Freeth, D., \&Zwarenstein, M. (2013). Interprofessional education: effects on professional practice and healthcare outcomes (update). Cochrane Database of Systematic Reviews, 3, 1-47.

Ritchie, C., Dann, L.\& Ford, P. (2013). Shared learning for oral health therapy and dental students: enhanced understanding of roles and responsibilities through interprofessional education. European Journal of Dental Education, 17(1), 5661.

Scherer, Y.K., Myers, J., O’Connor, T.D., \&Haskins, M. (2013). Interprofessional simulation to foster collaboration between nursing and medical students. Clinical Simulation in Nursing, 9(11), 497-505.

Takahashi, H. E. (2007). Interprofessional education needs to correspond with the necessity of interprofessional work in health-care and welfare in Japan. The Niigata Journal of Health and Welfare, 7(1), 1-8.

Thistlethwaite, J., \& Moran, M. (2010). Learning outcomes for interprofessional education (IPE): literature review and synthesis. Journal of Interprofessional Care, 24(5), 503-515.

WHO.(1988). Learning together to work together for health. Report of a WHO Study Group on Multiprofessional Education for Health Personnel: The Team Approach. World Health Organization, Geneva. 